\title{
Influence of selenate and linseed oil on fatty-acid and amino-acid profiles in the liver, muscles, fat tissues and blood plasma of sheep
}

\author{
K.M. Niedźwiedzka, J. Kowalczyk and M. Czauderna ${ }^{1}$ \\ The Kielanowski Institute of Animal Physiology and Nutrition, Polish Academy of Sciences \\ 05-110 Jabtonna, Poland
}

(Received 7 May 2008; accepted 24 June 2008)

\begin{abstract}
Twenty individually-housed male Polish Merino lambs with an initial body weight of $25 \pm 2 \mathrm{~kg}$ were used in the experiment. Each group comprised 5 lambs. Sheep were fed the control or experimental diets enriched in 5\% linseed oil (LO), 2 ppm selenium as selenate (SE) or combined addition of 5\% LO and 2 ppm selenium (LOSE). The effects of the experimental diets on organs and body weight gain (BWG) of the sheep were small, but the LOSE treatment increased BWG and the weight of M. biceps femoris, M. longissimus dorsi, and the liver. The diets enriched in LO with or without SE decreased the concentration of atherogenic and thrombogenic saturated fatty acids in muscles, liver and perirenal fat tissues. The LO treatment increased the concentration of conjugated linoleic acid (CLA) isomers in the subcutaneous and perirenal fat tissues, liver, M. longissimus dorsi and blood plasma in comparison with the control. Dietary LO with or without SE resulted in negligible changes in the concentration of CLA isomers in M. biceps femoris. The diet enriched in SE decreased the concentration of CLA isomers in both muscles compared with control muscles, whereas it increased in the plasma, liver and perirenal fat tissue. There was a LO and SE interaction for the CLA isomer concentration in the perirenal fat tissue and plasma; this treatment resulted in an increase of the CLA isomer concentration in these tissues compared with the control. The diets enriched in LO or with LO and SE increased the concentration of polyunsaturated fatty acids (PUFA) in the subcutaneous fat tissue, plasma, and perirenal fat tissue. Feeding the diet with LO increased the concentrations of cis 9 cis $12 \mathrm{C} 18: 2$ and cis 9 cis 12 cis $15 \mathrm{C} 18: 3$ in the subcutaneous fat tissue, muscles and plasma in comparison with the control. LO supplementation to sheep increased the concentration of essential ( $\Sigma \mathrm{E}-\mathrm{AA})$, non-essential ( $\Sigma \mathrm{NE}-\mathrm{AA})$ and sulphur ( $\Sigma$ S-AA) amino acids in the muscles; the diet enriched in LO with or without SE decreased $\Sigma E-A A$, $\Sigma$ NE-AA, $\Sigma$ S-AA and $\Sigma$ AA in the liver. These diets increased the nutritional value of meats as the ratio of SFA/MUFA and SFA/PUFA decreased, while the concentration of $\Sigma$ E-AA increased. The diet containing SE with or without LO resulted in an increase in the Se concentration in meat, plasma, and liver compared with the control.
\end{abstract}

KEY WORDS: linseed oil, selenate, fatty acid, amino acid, muscle, liver, fat tissue, blood plasma, sheep

${ }^{1}$ Corresponding author: e-mail: m.czauderna@ifzz.pan.pl 


\section{INTRODUCTION}

The purpose of modifying the fatty acid composition of meat or milk is to produce high-quality products meeting dietary recommendations for increased intake of polyunsaturated fatty acids (PUFA), especially n-3PUFA, and reduced intake of atherogenic and thrombogenic saturated fatty acids (A-SFA and T-SFA). Indeed, many epidemiological and experimental studies (Niedźwiedzka et al., 2006; Murphy et al., 2007) documented that A-SFA (C12:0, C14:0 and C16:0) and T-SFA (C14:0, C16:0 and C18:0) possessed atherogenic and thrombogenic properties, while $\alpha$-linolenic acid and its products of elongation and desaturation (i.e. long-chain n3PUFA) improved anti-inflammatory status, immune response, as well as benefited the cardiovascular system by decreasing platelet aggregation, serum triglycerides and cholesterol (Raes et al., 2004; Flachowsky et al., 2006). On the other hand, a higher dietary content of n-6PUFA results in a pro-inflammatory status, therefore, it is recommended to maintain the n-6PUFA/n-3PUFA ratio below 4. Recent investigations documented that elevating the PUFA content in a diet stimulated oxidative deterioration in meat of ruminants and monogastric animals. Considering these arguments, an adequate concentration of n-3PUFA in diets together with antioxidants, like selenium, is essential for the good health of domestic animals and humans.

Diverse inorganic Se compounds, like selenate or selenite, are used as nutritional sources of Se. These compounds are metabolized to intermediates and then utilized

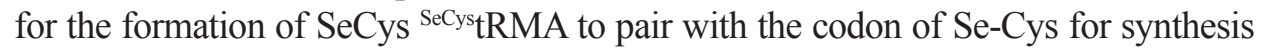
of proteins containing Se-Cys (e.g., the glutathione peroxidase family, selenoprotein P, or thioredoxin reductase). The principal physiological roles of half of Se-proteins are to maintain the appropriate metabolism of arachidonic acid and low concentrations of pre-oxides or free radicals within cells, thus decreasing oxidative stress in living organisms (Tapiero et al., 2003; Shweizer et al., 2005). Glutathione peroxidases (GPx) in particular provide a second line of defense against hydroperoxides, which can damage lipids in membranes as well as other cell structures. GSH-Px acts synergistically with tocopherol in the regulation of lipid peroxidation. Recent studies documented that phospholipid hydroperoxide GPx, in particular, interacted more directly than cytosolic and mitochondrial GPx in protecting PUFA from peroxidation damage (Crespo et al., 1995; Tapiero et al., 2003). A positive correlation was observed between concentrations of unsaturated FA and the dietary content of Se (Crespo et al., 1995; Tanguy et al., 2003; Yu et al., 2008).

Considering the above, we hypothesized that dietary inorganic Se and a vegetable oil rich in n-3PUFA would improve the nutritive properties of food derived from domestic ruminants. Therefore, the aim of our pilot investigations was to study the influence of a diet enriched in linseed oil (as a precursor of FA 
containing conjugated double bonds) and selenate (Se) on the profile of fatty acids (FA) and amino acids (AA) in muscles, liver, fat tissues and blood plasma of sheep.

\section{MATERIAL AND METHODS}

\section{Animals and diets}

Twenty male Polish Merino lambs with an average initial body weight (BW) of $25 \pm 2 \mathrm{~kg}$ were allotted to 4 groups of 5 animals and housed individually. During a 1-week preliminary period the animals were given ad libitum access to the standard concentrate-hay diet (Czauderna et al., 2004a,b). This diet contains, g: crude protein 120, crude fibre 120 and $11 \mathrm{MJ}$ metabolizable energy in $1 \mathrm{~kg}$ DM. The concentration of Se in the standard concentrate-hay diet was $0.1 \mathrm{ppm}$. After a 1 -week preliminary period, the lambs were fed for 35 days the standard concentrate-hay diet (Control), the experimental diets enriched in either $5 \%$ linseed oil (LO) or $2 \mathrm{ppm}$ selenium as sodium selenate (SE), or the experimental diet with the combined addition of 5\% LO and $2 \mathrm{ppm}$ selenium (LOSE). The fatty acid profile of LO is shown in Table 1. The rations were fed ad libitum.

Table 1 . The fatty acid composition in linseed oil

\begin{tabular}{lc}
\hline Fatty acids & Concentration, $\mu \mathrm{g} / \mathrm{g}$ \\
\hline C8:0 & 26.0 \\
C10:0 & 35.3 \\
C12:0 & 9.62 \\
C14:0 & 18.0 \\
C16:0 & 1405 \\
C18:0 & 872 \\
C18:3n-3 & 5892 \\
C18:3n-6 & 894 \\
C18:2n-6 & 4962 \\
c9C18:1 & 4169 \\
c6C18:1 & 539 \\
CLA isomers ${ }^{1}$ & 109 \\
SFA & 2413 \\
MUFA & 4718 \\
PUFA & 11749 \\
FA &
\end{tabular}

the concentration sum of: ${ }^{1} \mathrm{CLA}$ isomers, ${ }^{2}$ all assayed fatty acids (FA) 


\section{Chemicals}

All of the chemicals used for saponification of samples, solvents used for eluting, and Waters HPLC equipment (USA) were as described previously (Czauderna and Kowalczyk, 2002). A CLA isomer mixture (95-97\%), and isomers cis9, trans 11 (c9t11CLA) (95-97\%) and trans 10, cis 12 (t10,c12CLA) (95-97\%) were supplied by Larodan Fine Chemicals AB (Sweden). Other fatty acid standards and sodium selenate $\left(\mathrm{Na}_{2} \mathrm{SeO}_{4}\right)$ were provided by Sigma (USA).

Underivatized conjugated linoleic acid (CLA) isomers and other fatty acids containing conjugated double bonds (CFA) in hydrolysates were determined directly according to Czauderna et al. (2003a). Other FA as well as CLA isomers and CFA were derivatized and then quantified according to Czauderna et al. (2002b). Amino acid concentrations in all assayed samples were determined according to Czauderna et al. (2002a).

Amino acid concentrations in muscles and livers were analysed in pooled samples prepared by combination of all livers, M. biceps femoris and M. longissimus dorsi from sheep fed the same diet.

\section{Statistical analysis}

Statistical analyses of the effects of dietary Se or LO on fatty acid concentrations in sheep tissues and amino acids in blood plasma were conducted using the nonparametric Mann-Whitney $U$ test for comparing independent experimental groups, while statistical analyses of interactions between LO and Se were performed using two-factorial ANOVA analysis (LO x Se). Statistica ver. 6 and Microsoft Office Excel software were used (Statistica, 2002). Differences were considered significant at $\mathrm{P}<0.05$.

\section{RESULTS}

Dietary administration of LO and/or SE resulted in no lesions or symptoms of harmful effects in sheep fed the experimental diets. Experimental data on feed intake, body weight gain (BWG), feed conversion efficiency (FCE), weight of the liver, M. biceps femoris and M. longissimus dorsi after 35 days of feeding the experimental diets are summarized in Table 2. Results showed that feeding sheep the diet supplemented with LO slightly increased $(\mathrm{P}>0.05)$ the weight of $M$. biceps femoris and $M$. longissimus dorsi as well as BWG and FCE and consequently decreased feed intake. There was an OL x SE interaction $(\mathrm{P}=0.039)$ because sheep fed the diet enriched in OL and SE had increased BWG $(\mathrm{P}=0.039)$ and liver $(\mathrm{P}=0.049)$ and 
M. biceps femoris $(\mathrm{P}=0.043)$ weights. On the other hand, only minute effects of the extra selenate in the diet (SE) on feed intake, FCE, BWG and weight of both muscles were observed, whereas this diet resulted in a more distinctive but not statistically significant decrease in liver weight.

Table 2. Effects of diets on the feed intake, body weight gain (BWG), the conversion efficiency ${ }^{1}$ (FCE) and masses of the liver, M. biceps femoris and M. longissimus dorsi of sheep

\begin{tabular}{|c|c|c|c|c|c|c|c|c|c|}
\hline \multirow{4}{*}{ Item } & \multicolumn{4}{|c|}{ Group } & \multicolumn{5}{|c|}{ ANOVA } \\
\hline & \multirow{3}{*}{ control } & \multirow{3}{*}{$\mathrm{SE}^{2}$} & \multirow{3}{*}{$\mathrm{LO}^{3}$} & \multirow{3}{*}{$\mathrm{LO}+\mathrm{SE}^{4}$} & \multicolumn{4}{|c|}{ statistical effects } & \multirow{3}{*}{$\begin{array}{c}\begin{array}{c}\text { interaction } \\
\mathrm{OL} \times \mathrm{SE}\end{array} \\
\mathrm{P}\end{array}$} \\
\hline & & & & & \multicolumn{2}{|c|}{ SE } & \multicolumn{2}{|c|}{$\mathrm{LO}$} & \\
\hline & & & & & SEM & $\mathrm{P}$ & SEM & $\mathrm{P}$ & \\
\hline Feed intake, $\mathrm{kg}$ & 39.0 & 38.9 & 37.1 & 37.6 & 0.41 & NS & 0.46 & 0.03 & NS \\
\hline BWG, kg & $6.6^{\mathrm{a}}$ & $6.9^{\mathrm{a}}$ & $9.5^{\mathrm{b}}$ & $9.2^{\mathrm{ab}}$ & 1.8 & NS & 1.1 & NS & 0.039 \\
\hline $\mathrm{FCE}$ & 0.166 & 0.178 & 0.255 & 0.244 & 0.021 & NS & 0.020 & NS & NS \\
\hline Liver, $g$ & $618^{\mathrm{ab}}$ & $563^{\mathrm{a}}$ & $625^{\mathrm{ab}}$ & $659^{\mathrm{ab}}$ & 52 & NS & 31 & NS & 0.049 \\
\hline M. biceps femoris, $\mathrm{kg}$ & 4.1 & 4.3 & 4.7 & 4.6 & 205 & NS & 166 & NS & 0.043 \\
\hline M. longissimus dorsi, & 371 & 373 & 417 & 385 & 47 & NS & 36 & NS & NS \\
\hline
\end{tabular}

${ }^{1} \mathrm{~kg}$ body weight gain $/ \mathrm{kg}$ feed intake; sheep group fed diet enriched in: ${ }^{2}$ selenate (2 ppm Se), ${ }^{3} 5 \%$ linseed oil (w/w), ${ }^{4}$ selenate ( 2 ppm Se) and 5\% linseed oil (w/w); NS - P >0.05

The influence of dietary LO and SE on the SFA concentrations in tissues of sheep. The current study also investigated the relationship between the experimental diets and the saturated fatty acids (SFA) in assayed tissues of sheep (Tables 3, 4 and 5). In both muscles, the perirenal fat tissue and the liver, A-SFA

Table 3. Effects of the diets enriched in linseed oil (LO) and/or selenate (SE) on the concentration $(\mathrm{mg} / \mathrm{g})$ of fatty acids (FA) and amino acids (AA) in M. biceps femoris of sheep ${ }^{1}$

\begin{tabular}{|c|c|c|c|c|c|c|c|c|c|}
\hline \multirow{3}{*}{ Fatty acids } & \multirow{2}{*}{\multicolumn{4}{|c|}{ Group }} & \multicolumn{4}{|c|}{ Statistical effect ${ }^{9}$} & \multirow{3}{*}{$\begin{array}{c}\begin{array}{c}\text { Inter- } \\
\text { action } \\
\mathrm{OL} \times \mathrm{SE}\end{array} \\
\mathrm{P}\end{array}$} \\
\hline & & & & & \multicolumn{2}{|l|}{$\mathrm{SE}$} & \multicolumn{2}{|c|}{ LO } & \\
\hline & control & $\mathrm{SE}$ & $\mathrm{LO}$ & $\mathrm{LO}+\mathrm{SE}$ & SEM & $\mathrm{P}$ & SEM & $\mathrm{P}$ & \\
\hline \multicolumn{10}{|c|}{ M. biceps femoris } \\
\hline A-SFA & $1.93^{\mathrm{a}}$ & $1.24^{\mathrm{c}}$ & $1.35^{\mathrm{bc}}$ & $1.71^{\mathrm{ab}}$ & 0.12 & NS & 0.104 & NS & 0.002 \\
\hline T-SFA & $3.04^{\mathrm{a}}$ & $2.01^{\mathrm{c}}$ & $2.22^{\mathrm{bc}}$ & $2.81^{\mathrm{ab}}$ & 0.18 & NS & 0.158 & NS & 0.003 \\
\hline $\mathrm{LA}^{2}$ & $1.34^{\mathrm{cb}}$ & $1.11^{\mathrm{b}}$ & $1.60^{\mathrm{ab}}$ & $1.89^{\mathrm{a}}$ & 0.13 & NS & 0.114 & 0.008 & NS \\
\hline$\gamma$-LNA ${ }^{2}$ & $0.001^{\mathrm{a}}$ & $0.001^{\mathrm{a}}$ & $0.002^{\mathrm{a}}$ & $0.001^{\mathrm{a}}$ & 0.0002 & NS & 0.0003 & NS & NS \\
\hline$\alpha-\mathrm{LNA}^{2}$ & $0.20^{\mathrm{b}}$ & $0.14^{\mathrm{b}}$ & $0.23^{\text {ba }}$ & $0.35^{\mathrm{a}}$ & 0.03 & NS & 0.025 & 0.001 & 0.016 \\
\hline $\mathrm{CLA}^{3}$ & $0.35^{\mathrm{a}}$ & $0.18^{\mathrm{b}}$ & $0.31^{\mathrm{a}}$ & $0.31^{\mathrm{a}}$ & 0.03 & NS & 0.03 & NS & NS \\
\hline $\mathrm{CFA}^{3}$ & $0.116^{\mathrm{ab}}$ & $0.091^{\mathrm{a}}$ & $0.073^{\mathrm{a}}$ & $0.156^{\mathrm{b}}$ & 0.016 & NS & 0.015 & NS & NS \\
\hline$\Sigma t \mathrm{C} 18: 1^{4}$ & $0.119^{\mathrm{ab}}$ & $0.063^{\mathrm{c}}$ & $0.072^{\mathrm{ac}}$ & $0.138^{\mathrm{b}}$ & 0.013 & NS & 0.012 & NS & 0.003 \\
\hline$c 9 \mathrm{C} 18: 1$ & $3.02^{\mathrm{a}}$ & $1.92^{\mathrm{b}}$ & $1.76^{\mathrm{b}}$ & $2.42^{\mathrm{ab}}$ & 0.19 & NS & 0.19 & NS & 0.002 \\
\hline SFA/MUFA ${ }^{5}$ & $0.52^{\mathrm{b}}$ & $0.63^{\mathrm{a}}$ & $0.38^{\mathrm{c}}$ & $0.42^{\mathrm{c}}$ & 0.03 & 0.02 & 0.02 & 0.001 & NS \\
\hline SFA/PUFA ${ }^{5}$ & $1.99^{\mathrm{a}}$ & $1.62^{\mathrm{b}}$ & $1.22^{\mathrm{c}}$ & $1.25^{\mathrm{c}}$ & 0.10 & NS & 0.11 & 0.001 & 0.046 \\
\hline
\end{tabular}


Table 3 continued

\begin{tabular}{|c|c|c|c|c|c|c|c|c|c|}
\hline \multirow{3}{*}{ Fatty acids } & \multirow{2}{*}{\multicolumn{4}{|c|}{ Group }} & \multicolumn{4}{|c|}{ Statistical effect ${ }^{9}$} & \multirow{3}{*}{$\begin{array}{c}\begin{array}{c}\text { Inter- } \\
\text { action } \\
\mathrm{OL} \times \mathrm{SE}\end{array} \\
\mathrm{P}\end{array}$} \\
\hline & & & & & \multicolumn{2}{|c|}{ SE } & \multicolumn{2}{|l|}{$\mathrm{LO}$} & \\
\hline & control & SE & LO & $\mathrm{LO}+\mathrm{SE}$ & SEM & $\mathrm{P}$ & SEM & $\mathrm{P}$ & \\
\hline$\Sigma \mathrm{FA}^{5}$ & $11.1^{\mathrm{a}}$ & $6.82^{\mathrm{b}}$ & $10.5^{\mathrm{ab}}$ & $12.8^{\mathrm{a}}$ & 0.98 & NS & 0.77 & NS & 0.020 \\
\hline$\sum \mathrm{E}^{-\mathrm{AA}^{6}}$ & 298 & 320 & 312 & 293 & - & - & - & - & - \\
\hline$\sum \mathrm{NE}^{-\mathrm{AA}^{6}}$ & 308 & 358 & 321 & 327 & - & - & - & - & - \\
\hline cysteine & 14.2 & 15.4 & 16.3 & 15.9 & - & - & - & - & - \\
\hline taurine & 56 & 61 & 62 & 55 & - & - & - & - & - \\
\hline methionine & 7.23 & 11.57 & 7.27 & 8.98 & - & - & - & - & - \\
\hline lysine & 51 & 57 & 56 & 50 & - & - & - & - & - \\
\hline$\sum \mathrm{S}-\mathrm{AA}^{7}$ & 77 & 88 & 86 & 80 & & & & & \\
\hline$\sum \mathrm{AA}^{6}$ & 606 & 678 & 633 & 621 & - & - & - & - & - \\
\hline \multicolumn{10}{|c|}{ M. longissimus dorsi } \\
\hline A-SFA & $2.43^{\mathrm{a}}$ & $1.67^{\mathrm{ab}}$ & $1.70^{\mathrm{b}}$ & $1.53^{\mathrm{b}}$ & 0.19 & NS & 0.17 & NS & NS \\
\hline T-SFA & $3.80^{\mathrm{a}}$ & $2.74^{\mathrm{b}}$ & $2.75^{b}$ & $2.55^{\mathrm{b}}$ & 0.26 & NS & 0.25 & NS & NS \\
\hline LA & $1.06^{\mathrm{a}}$ & $0.93^{\mathrm{a}}$ & $1.12^{\mathrm{a}}$ & $0.79^{\mathrm{a}}$ & 0.07 & 0.045 & 0.07 & NS & NS \\
\hline$\gamma$-LNA & 0.001 & -8 & 0.001 & -8 & 0.0002 & NS & 0.0002 & NS & NS \\
\hline$\alpha$-LNA & $0.13^{\mathrm{a}}$ & $0.11^{\mathrm{a}}$ & $0.15^{\mathrm{a}}$ & $0.11^{\mathrm{a}}$ & 0.01 & NS & 0.01 & NS & NS \\
\hline CLA & $0.35^{\mathrm{ab}}$ & $0.20^{c}$ & $0.40^{\mathrm{a}}$ & $0.24^{\mathrm{bc}}$ & 0.03 & 0.007 & 0.033 & NS & NS \\
\hline CFA & $0.107^{\mathrm{a}}$ & $0.067^{\mathrm{ab}}$ & $0.066^{\mathrm{b}}$ & $0.079^{\mathrm{ab}}$ & 0.011 & NS & 0.012 & NS & NS \\
\hline$\Sigma t \mathrm{C} 18: 1$ & $0.109^{\mathrm{a}}$ & $0.090^{\mathrm{ab}}$ & $0.096^{\mathrm{ab}}$ & $0.091^{\mathrm{b}}$ & 0.008 & NS & 0.007 & NS & NS \\
\hline$c 9 \mathrm{C} 18: 1$ & $3.05^{\mathrm{a}}$ & $2.28^{\mathrm{ab}}$ & $2.11^{\mathrm{b}}$ & $1.87^{\mathrm{ab}}$ & 0.25 & NS & 0.24 & NS & NS \\
\hline SFA/MUFA & $0.67^{\mathrm{a}}$ & $0.70^{\mathrm{ab}}$ & $0.37^{\mathrm{b}}$ & $0.46^{\mathrm{b}}$ & 0.04 & NS & 0.038 & 0.001 & NS \\
\hline SFA/PUFA & $3.16^{\mathrm{a}}$ & $2.69^{\mathrm{ab}}$ & $2.16^{\mathrm{b}}$ & $2.91^{\mathrm{ab}}$ & 0.15 & NS & 0.159 & NS & 0.019 \\
\hline$\Sigma \mathrm{FA}$ & $11.47^{\mathrm{a}}$ & $8.16^{\mathrm{a}}$ & $12.14^{\mathrm{a}}$ & $9.52^{\mathrm{a}}$ & 0.81 & 0.03 & 0.78 & NS & NS \\
\hline$\sum \mathrm{E}-\mathrm{AA}$ & 267 & 307 & 284 & 282 & - & - & - & - & - \\
\hline$\sum \mathrm{NE}-\mathrm{AA}$ & 307 & 347 & 323 & 315 & - & - & - & - & - \\
\hline cysteine & 2.46 & 8.45 & 9.34 & 8.96 & - & - & - & - & - \\
\hline taurine & 54 & 60 & 57 & 55 & - & - & - & - & - \\
\hline methionine & 12.57 & 15.46 & 14.22 & 12.60 & - & - & - & - & - \\
\hline lysine & 53 & 58 & 52 & 53 & - & - & - & - & - \\
\hline$\sum \mathrm{S}-\mathrm{AA}$ & 69 & 84 & 81 & 77 & - & - & - & - & - \\
\hline$\sum \mathrm{AA}$ & 574 & 654 & 608 & 597 & - & - & - & - & - \\
\hline
\end{tabular}

${ }^{1}$ means in rows with different letters are significantly different at the ${ }^{\mathrm{a}, \mathrm{b}} \mathrm{P}<0.05 ;{ }^{2} \mathrm{LA}$ - linoleic acid; $\alpha$-LNA - $\alpha$-linolenic acid; $\gamma$-LNA - $\gamma$-linolenic acid; ${ }^{3}$ CLA - the sum of CLA isomers; CFA - nonCLA conjugated fatty acids; ${ }^{4} \Sigma t \mathrm{C} 18: 1$ - the sum of $t 6 \mathrm{C} 18: 1, t 7 \mathrm{C} 18: 1, t 9 \mathrm{C} 18: 1$ and $t 11 \mathrm{C} 18: 1 ;{ }^{5}$ SFAMUFA, SFA/PUFA and $\Sigma F A$ - the ratio of SFA to MUFA, the ratio of SFA to PUFA and the sum of all FA, respectively; ${ }^{6}$ the sum of essential ( $\left.\sum \mathrm{E}-\mathrm{AA}\right)$, non-essential ( $\left.\sum \mathrm{NE}-\mathrm{AA}\right)$ and all amino acids $\left(\sum \mathrm{AA}\right) ;{ }^{7}$ the sum of methionine, cysteine and taurine; ${ }^{8}$ below the quantification limit $\left(\mathrm{L}_{\mathrm{Q}}\right) ;{ }^{9} \mathrm{NS}-\mathrm{P}>0.05$ 
and T-SFA concentrations decreased (usually statistically significantly; $\mathrm{P}<0.05$ ) with the addition of LO to the diet. Concomitantly with this, acid concentrations decreased, values of the concentration ratios of SFA/MUFA and SFA/PUFA also decreased $(\mathrm{P}<0.05)$ in these sheep tissues. Similarly, the diet with extra selenate resulted in a decrease of the A-STA and T-SFA in M. biceps femoris $(\mathrm{P}<0.05)$, but usually statistically non-significantly in M. longissimus dorsi and the liver. Feeding sheep the diet enriched in LO resulted in an increase $(\mathrm{P}<0.05)$ in the concentrations of SFA, A-SFA and T-SFA in the subcutaneous fat tissue and blood plasma, whereas changes of values of the concentration ratios of SFA/MUFA and SFA/PUFA in these tissues were inconsistent with changes of the concentrations of SFA, A-SFA and T-SFA; the ratios were usually lower in comparison with those of these fatty acids in the control (Tables 4 and 5). The diet with extra selenate resulted in an increase $(\mathrm{P}<0.05)$ in the concentration of A-SFA and T-SFA in blood plasma and subcutaneous fat tissues.

There were $\mathrm{LO} \times \mathrm{SE}$ interaction effects $(\mathrm{P}<0.01)$ on the concentrations of A-SFA and T-SFA in M. biceps femoris and blood plasma. The simultaneous addition of $\mathrm{LO}$ and SE to the diet decreased the A-SFA and T-SFA concentrations in the liver $(\mathrm{P}<0.05)$, M. longissimus dorsi $(\mathrm{P}<0.05)$, while only slightly decreased the concentration of these acids in M. biceps femoris and the perirenal fat tissue in comparison with the concentrations in the control. This diet resulted in an increase of the SFA, A-SFA and T-SFA concentration in the subcutaneous fat tissue $(\mathrm{P}<0.05)$, but statistically non-significant in blood plasma compared with the control group.

The influence of dietary LO and SE on the concentrations of CLA isomers and $C F A$ in tissues of sheep. The LO treatment increased $(\mathrm{P}<0.05)$ the concentration of CLA isomers in both the fat tissues and liver, whereas it insignificantly elevated it in M. longissimus dorsi and blood plasma in comparison with the control tissues. On the other hand, dietary LO, regardless of the presence of extra selenate in the diet, resulted in negligible changes in the concentration of CLA isomers in $M$. biceps femoris. The diet enriched in only SE decreased $(\mathrm{P}<0.05)$ the concentration of CLA isomers in both muscles compared with the control, whereas increased $(\mathrm{P}<0.05)$ it in the blood plasma, liver and, insignificantly, in the perirenal fat tissue $(\mathrm{P}=0.02)$. There was a $\mathrm{LO}$ and $\mathrm{SE}$ interaction for the CLA isomer concentration in the perirenal fat tissue $(\mathrm{P}=0.03)$ and blood plasma $(\mathrm{P}=0.04)$; this treatment resulted in an increase of the CLA isomer concentration in these tissues compared with the control.

The effect of dietary LO and/or SE on the CFA concentration was analysed in the assayed tissues of sheep. The LA supplement decreased $(\mathrm{P}<0.05)$ the CFA concentration in $M$. longissimus dorsi and tended to decrease it in M. biceps femoris $(\mathrm{P}=0.07)$, whereas it increased $(\mathrm{P}<0.05)$ it in the subcutaneous fat tissue compared 
Table 4. Effects of the diets enriched in linseed oil (LO) and/or selenate (SE) on the concentration $(\mathrm{mg} / \mathrm{g})$ of fatty acids (FA) and amino acids (AA) in the liver and subcutaneous fat tissues of sheep ${ }^{1}$

\begin{tabular}{|c|c|c|c|c|c|c|c|c|c|}
\hline \multirow{3}{*}{ Fatty acids ${ }^{2}$} & \multirow{2}{*}{\multicolumn{4}{|c|}{ Group }} & \multicolumn{4}{|c|}{ Statistical effect ${ }^{4}$} & \multirow{3}{*}{$\begin{array}{c}\begin{array}{c}\text { Inter- } \\
\text { action }\end{array} \\
\mathrm{OL} \times \mathrm{SB} \\
\mathrm{P}\end{array}$} \\
\hline & & & & & \multicolumn{2}{|c|}{ SE } & \multicolumn{2}{|c|}{ LO } & \\
\hline & control & SE & LO & $\mathrm{LO}+\mathrm{SE}$ & SEM & $\mathrm{P}$ & SEM & $\mathrm{P}$ & \\
\hline \multicolumn{10}{|l|}{ Liver } \\
\hline A-SFA & $5.80^{\mathrm{a}}$ & $5.56^{\mathrm{a}}$ & $3.20^{\mathrm{b}}$ & $2.78^{\mathrm{b}}$ & 0.43 & NS & 0.42 & 0.001 & NS \\
\hline T-SFA & $12.4^{\mathrm{a}}$ & $11.0^{\mathrm{ab}}$ & $8.2^{\mathrm{ab}}$ & $6.60^{\mathrm{b}}$ & 1.1 & NS & 1.0 & 0.007 & NS \\
\hline LA & $2.91^{\mathrm{a}}$ & $3.08^{\mathrm{a}}$ & $3.98^{\mathrm{b}}$ & $3.15^{\mathrm{a}}$ & 0.18 & NS & 0.32 & NS & NS \\
\hline$\gamma$-LNA & $0.11^{\mathrm{a}}$ & $0.13^{\mathrm{a}}$ & $0.68^{\mathrm{b}}$ & $0.63^{\mathrm{b}}$ & 0.08 & NS & 0.09 & 0.001 & NS \\
\hline$\alpha$-LNA & $0.32^{\mathrm{ab}}$ & $0.39^{\mathrm{a}}$ & $0.25^{\mathrm{b}}$ & $0.25^{\mathrm{b}}$ & 0.03 & NS & 0.03 & NS & NS \\
\hline CLA & $1.04^{\mathrm{a}}$ & $1.73^{\mathrm{b}}$ & $1.57^{\mathrm{b}}$ & $0.92^{\mathrm{a}}$ & 0.19 & NS & 0.18 & NS & NS \\
\hline CFA & $2.40^{\mathrm{a}}$ & $0.37^{b}$ & $2.36^{\mathrm{a}}$ & $0.84^{b}$ & 0.28 & 0.003 & 0.37 & NS & NS \\
\hline$\Sigma t \mathrm{C} 18: 1$ & $0.39^{a}$ & $0.54^{\mathrm{ab}}$ & $0.45^{\mathrm{b}}$ & $0.39^{\mathrm{a}}$ & 0.03 & NS & 0.02 & NS & 0.04 \\
\hline C9C18:1 & $6.12^{\mathrm{a}}$ & $5.70^{\mathrm{ab}}$ & $4.25^{\mathrm{b}}$ & $4.15^{\mathrm{b}}$ & 0.39 & NS & 0.37 & 0.006 & NS \\
\hline SFAMUFA & $0.58^{\mathrm{a}}$ & $0.62^{\mathrm{a}}$ & $0.17^{b}$ & $0.19^{b}$ & 0.07 & NS & 0.06 & 0.001 & NS \\
\hline SFAPUFA & $3.54^{\mathrm{a}}$ & $2.61^{\mathrm{b}}$ & $1.78^{\mathrm{b}}$ & $1.42^{\mathrm{b}}$ & 0.31 & NS & 0.34 & 0.001 & NS \\
\hline$\Sigma \mathrm{FA}$ & $41.9^{\mathrm{a}}$ & $37.1^{\mathrm{a}}$ & $66.9^{\mathrm{b}}$ & $49.2^{\mathrm{a}}$ & 2.9 & 0.034 & 3.7 & 0.001 & NS \\
\hline$\sum \mathrm{E}-\mathrm{AA}$ & 270 & 282 & 242 & 249 & - & - & - & - & - \\
\hline$\sum \mathrm{NE}-\mathrm{AA}$ & 281 & 298 & $248 \quad 2$ & 262 & - & - & - & - & - \\
\hline cysteine & 6.45 & 3.45 & 3.55 & 3.30 & - & - & - & - & - \\
\hline taurine & 41 & 45 & 38 & 42 & - & - & - & - & - \\
\hline methionine & 9.15 & 4.73 & 5.09 & 4.72 & - & - & - & - & - \\
\hline lysine & 42 & 45 & 38 & 40 & - & - & - & - & - \\
\hline$\sum \mathrm{S}-\mathrm{AA}$ & 57 & 53 & 47 & 50 & - & - & - & - & - \\
\hline$\sum \mathrm{AA}$ & 550 & 580 & 490 & 511 & - & - & - & - & - \\
\hline \multicolumn{10}{|c|}{ Subcutaneous fat tissue } \\
\hline SFA & $9.89^{\mathrm{b}}$ & $26.8^{\mathrm{a}}$ & $29.8^{\mathrm{a}}$ & $32.7^{\mathrm{a}}$ & 3.2 & 0.03 & 3.6 & 0.01 & NS \\
\hline A-SFA & $7.02^{b}$ & $18.1^{\mathrm{a}}$ & $17.1^{\mathrm{a}}$ & $19.5^{\mathrm{a}}$ & 1.8 & 0.01 & 1.9 & 0.02 & NS \\
\hline T-SFA & $9.59^{\mathrm{b}}$ & $26.4^{\mathrm{a}}$ & $29.4^{\mathrm{a}}$ & $32.2^{\mathrm{a}}$ & 3.1 & 0.03 & 3.6 & 0.01 & NS \\
\hline LA & $1.34^{\mathrm{b}}$ & $6.77^{\mathrm{a}}$ & $7.39^{\mathrm{a}}$ & $9^{\mathrm{a}} \quad 8.07^{\mathrm{a}}$ & 1.0 & 0.04 & 1.12 & 0.02 & NS \\
\hline$\gamma$-LNA & $0.14^{\mathrm{a}}$ & $0.12^{\mathrm{a}}$ & $0.11^{\mathrm{a}}$ & $0.12^{\mathrm{a}}$ & 0.01 & NS & 0.01 & NS & NS \\
\hline$\alpha$-LNA & $5.12^{\mathrm{a}}$ & $2.91^{\mathrm{a}}$ & $8.57^{\mathrm{b}}$ & $9.47^{b}$ & 0.95 & NS & 1.03 & 0.003 & NS \\
\hline CLA & $4.79^{\mathrm{a}}$ & $4.26^{\mathrm{a}}$ & $6.11^{\mathrm{b}}$ & $5.72^{\mathrm{ab}}$ & 0.64 & NS & 0.70 & NS & NS \\
\hline CFA & $0.10^{\mathrm{b}}$ & $0.05^{\mathrm{b}}$ & $0.27^{\mathrm{a}}$ & $0.12^{\mathrm{b}}$ & 0.01 & 0.01 & 0.03 & 0.0004 & NS \\
\hline$\Sigma t \mathrm{C} 18: 1$ & $2.64^{\mathrm{c}}$ & $3.40^{\mathrm{b}}$ & $4.26^{\mathrm{a}}$ & $5^{\mathrm{a}} \quad 3.81^{\mathrm{ab}}$ & 0.48 & NS & 0.67 & 0.008 & 0.048 \\
\hline c9C18:1 & $26^{\mathrm{b}}$ & $37^{\mathrm{a}}$ & $33^{\mathrm{a}}$ & $44^{\mathrm{a}}$ & 3 & 0.04 & 5 & NS & NS \\
\hline MUFA & $77^{\mathrm{a}}$ & $84^{\mathrm{a}}$ & $169^{\mathrm{cb}}$ & $157^{\mathrm{b}}$ & 3 & 0.02 & 9 & 0.004 & NS \\
\hline PUFA & $6.77^{\mathrm{b}}$ & $9.94^{\mathrm{ab}}$ & $16.3^{\mathrm{a}}$ & $17.9^{\mathrm{a}}$ & 1.7 & NS & 2.1 & 0.006 & NS \\
\hline SFAMUFA & $0.62^{\mathrm{a}}$ & $0.26^{\mathrm{b}}$ & $0.22^{\mathrm{b}}$ & $2^{\mathrm{b}} \quad 0.21^{\mathrm{b}}$ & 0.05 & 0.001 & 0.05 & 0.001 & 0.001 \\
\hline SFAPUFA & $1.52^{\mathrm{b}}$ & $2.87^{\mathrm{a}}$ & $1.97^{\mathrm{b}}$ & $1.88^{\mathrm{b}}$ & 0.20 & 0.01 & 0.09 & NS & 0.006 \\
\hline$\Delta 9$-index ${ }^{3}$ & $0.75^{\mathrm{ab}}$ & $0.83^{\mathrm{a}}$ & $0.72^{\mathrm{b}}$ & $0.76^{\mathrm{ab}}$ & 0.02 & 0.046 & 0.02 & $\mathrm{NS}$ & NS \\
\hline
\end{tabular}

${ }^{1}$ means in rows with different letters are significantly different at ${ }^{\mathrm{a}, \mathrm{b}} \mathrm{P}<0.05$

2 abbreviations for fatty acids and other items see Table 1

${ }^{3}$ the $\Delta 9$ - desaturase index - the concentration ratio: $c i s-9 \mathrm{C} 18: 1 /(\mathrm{C} 18: 0+$ cis- $9 \mathrm{C} 18: 1)$

${ }^{4} \mathrm{NS}-\mathrm{P}>0.05$ 
Table 5. Effects of the diets enriched in linseed oil (LO) and/or selenate (SE) on the concentration (mg/g) of fatty acids (FA) and amino acids (AA) in the perirenal fat tissue and plasma blood of sheep ${ }^{1}$

\begin{tabular}{|c|c|c|c|c|c|c|c|c|c|}
\hline \multirow{3}{*}{ Fatty acids ${ }^{2}$} & \multirow{2}{*}{\multicolumn{4}{|c|}{ Group }} & \multicolumn{4}{|c|}{ Statistical effect ${ }^{5}$} & \multirow{3}{*}{$\begin{array}{c}\begin{array}{c}\text { Inter- } \\
\text { action }\end{array} \\
\mathrm{OL} \times \mathrm{SE} \\
\mathrm{P}\end{array}$} \\
\hline & & & & & \multicolumn{2}{|c|}{ SE } & \multicolumn{2}{|c|}{$\mathrm{LO}$} & \\
\hline & control & SE & $\mathrm{LO}$ & $\mathrm{LO}+\mathrm{SE}$ & SEM & $\mathrm{P}$ & SEM & $\mathrm{P}$ & \\
\hline \multicolumn{10}{|c|}{ Perirenal fat tissue } \\
\hline SFA & $23.3^{\mathrm{a}}$ & $22.9^{\mathrm{a}}$ & $17.3^{\mathrm{a}}$ & $17.6^{\mathrm{a}}$ & 1.4 & NS & 1.3 & 0.02 & NS \\
\hline A-SFA & $12.9^{\mathrm{a}}$ & $14.0^{\mathrm{a}}$ & $10.2^{\mathrm{ab}}$ & $8.89^{\mathrm{b}}$ & 0.91 & NS & 0.71 & 0.005 & NS \\
\hline T-SFA & $20.9^{\mathrm{a}}$ & $20.0^{\mathrm{a}}$ & $15.8^{\mathrm{b}}$ & $16.9^{\mathrm{a}}$ & 1.2 & NS & 1.2 & NS & NS \\
\hline LA & $2.90^{\mathrm{b}}$ & $4.70^{\mathrm{a}}$ & $3.00^{\mathrm{b}}$ & $4.10^{\mathrm{ab}}$ & 0.35 & 0.01 & 0.254 & NS & NS \\
\hline$\gamma$-LNA & $0.061^{\mathrm{a}}$ & $0.032^{\mathrm{b}}$ & $0.030^{\mathrm{b}}$ & $0.031^{\mathrm{b}}$ & 0.006 & NS & 0.006 & NS & NS \\
\hline$\alpha$-LNA & $0.34^{\mathrm{a}}$ & $0.20^{\mathrm{b}}$ & $0.16^{\mathrm{b}}$ & $0.19^{\mathrm{b}}$ & 0.03 & NS & 0.032 & 0.04 & NS \\
\hline CLA & $1.62^{\mathrm{b}}$ & $2.70^{\mathrm{ab}}$ & $3.05^{\mathrm{a}}$ & $2.41^{\mathrm{ab}}$ & 0.22 & NS & 0.23 & NS & 0.03 \\
\hline CFA & -3 & $0.037^{\mathrm{a}}$ & -3 & $0.306^{\mathrm{b}}$ & 0.04 & 0.001 & 0.04 & 0.001 & 0.01 \\
\hline$\Sigma t \mathrm{C} 18: 1$ & $1.59^{\mathrm{b}}$ & $2.33^{\mathrm{a}}$ & $1.64^{\mathrm{b}}$ & $1.82^{\mathrm{ab}}$ & 0.14 & 0.049 & 0.11 & NS & NS \\
\hline c9C18:1 & $17.3^{\mathrm{b}}$ & $31.4^{\mathrm{a}}$ & $13.5^{\mathrm{b}}$ & $19.5^{\mathrm{b}}$ & 2.3 & 0.001 & 1.341 & 0.01 & NS \\
\hline MUFA & $79^{\mathrm{a}}$ & $81^{\mathrm{a}}$ & $106^{\mathrm{b}}$ & $84^{\mathrm{ab}}$ & 7 & NS & 7 & NS & NS \\
\hline PUFA & $3.61^{\mathrm{a}}$ & $5.38^{\mathrm{a}}$ & $4.30^{\mathrm{a}}$ & $5.58^{\mathrm{a}}$ & 0.43 & 0.03 & 0.37 & NS & NS \\
\hline SFA/MUFA & $0.31^{\mathrm{a}}$ & $0.29^{\mathrm{ab}}$ & $0.16^{\mathrm{c}}$ & $0.22^{\mathrm{bc}}$ & 0.02 & NS & 0.02 & 0.001 & NS \\
\hline SFA/PUFA & $6.71^{\mathrm{a}}$ & $4.40^{\mathrm{b}}$ & $4.09^{\mathrm{b}}$ & $3.37^{\mathrm{b}}$ & 0.51 & 0.02 & 0.51 & 0.006 & NS \\
\hline$\Delta 9$-index & $0.63^{\mathrm{b}}$ & $0.78^{\mathrm{a}}$ & $0.65^{\mathrm{b}}$ & $0.69^{\mathrm{b}}$ & 0.02 & 0.001 & 0.014 & NS & 0.03 \\
\hline \multicolumn{10}{|l|}{ Blood plasma } \\
\hline SFA & $10.1^{\mathrm{c}}$ & $30.6^{\mathrm{a}}$ & $21.7^{\mathrm{b}}$ & $16.0^{\mathrm{cb}}$ & 2.6 & 0.01 & 1.8 & NS & 0.01 \\
\hline A-SFA & $5.17^{\mathrm{b}}$ & $15.1^{\mathrm{a}}$ & $8.20^{\mathrm{b}}$ & $6.48^{\mathrm{b}}$ & 1.31 & 0.002 & 0.63 & 0.03 & 0.01 \\
\hline T-SFA & $8.87^{\mathrm{c}}$ & $29.3^{\mathrm{a}}$ & $20.6^{\mathrm{b}}$ & $15.2^{\mathrm{c}}$ & 2.6 & 0.003 & 1.8 & NS & 0.01 \\
\hline LA & $2.23^{\mathrm{b}}$ & $7.14^{\mathrm{a}}$ & $6.08^{\mathrm{a}}$ & $3.73^{\mathrm{b}}$ & 0.68 & NS & 0.58 & NS & 0.01 \\
\hline$\gamma$-LNA & $0.07^{a}$ & $0.11^{\mathrm{ab}}$ & $0.28^{\text {bd }}$ & $0.13^{\mathrm{b}}$ & 0.01 & 0.01 & 0.05 & 0.001 & 0.01 \\
\hline$\alpha$-LNA & $0.50^{\mathrm{a}}$ & $0.52^{\mathrm{ab}}$ & $0.57^{\mathrm{b}}$ & $0.72^{\mathrm{b}}$ & 0.03 & NS & 0.04 & 0.001 & 0.01 \\
\hline CLA & $1.17^{b}$ & $4.74^{\mathrm{a}}$ & $2.32^{\mathrm{ab}}$ & $2.06^{\mathrm{ab}}$ & 0.66 & NS & 0.24 & NS & 0.04 \\
\hline CFA & -3 & -3 & -3 & -3 & - & - & - & - & - \\
\hline$\Sigma t \mathrm{C} 18: 1$ & $2.18^{\mathrm{b}}$ & $2.04^{b}$ & $9.60^{\mathrm{a}}$ & $6.57^{\mathrm{a}}$ & 0.78 & NS & 1.15 & 0.001 & NS \\
\hline c9C18:1 & $2.99^{b}$ & $10.2^{\mathrm{a}}$ & $4.88^{\mathrm{b}}$ & $3.95^{\mathrm{b}}$ & 1.10 & 0.02 & 0.41 & NS & 0.01 \\
\hline MUFA & $6.18^{\mathrm{a}}$ & $5.37^{\mathrm{a}}$ & $16.9^{\mathrm{b}}$ & $12.7^{\mathrm{b}}$ & 7.1 & 0.01 & 1.7 & NS & 0.01 \\
\hline PUFA & $2.83^{c}$ & $9.77^{\mathrm{ab}}$ & $12.3^{\mathrm{a}}$ & $7.42^{\mathrm{b}}$ & 0.98 & NS & 1.25 & 0.01 & 0.01 \\
\hline SFA/MUFA & $1.67^{\mathrm{a}}$ & $0.73^{\mathrm{b}}$ & $1.37^{\mathrm{a}}$ & $1.31^{\mathrm{a}}$ & 0.13 & 0.01 & 0.10 & NS & 0.02 \\
\hline SFA/PUFA & $3.71^{\mathrm{a}}$ & $3.18^{\mathrm{a}}$ & $1.77^{\mathrm{b}}$ & $2.31^{\mathrm{b}}$ & 0.19 & NS & 0.24 & 0.001 & 0.02 \\
\hline$\Delta 9$-index & $0.41^{\mathrm{a}}$ & $0.40^{\mathrm{a}}$ & $0.27^{\mathrm{b}}$ & $0.32^{\mathrm{ab}}$ & 0.02 & NS & 0.02 & 0.01 & NS \\
\hline$\Sigma F A$ & $26.1^{\mathrm{a}}$ & $92.9^{\mathrm{b}}$ & $53.2^{\mathrm{c}}$ & $82.6^{\mathrm{c}}$ & 10.1 & 0.003 & 4.3 & NS & 0.001 \\
\hline$\sum \mathrm{E}-\mathrm{AA}$ & $0.21^{\mathrm{a}}$ & $0.18^{\mathrm{a}}$ & $0.23^{\mathrm{a}}$ & $0.20^{\mathrm{a}}$ & 0.011 & NS & 0.014 & NS & NS \\
\hline$\sum \mathrm{NE}-\mathrm{AA}$ & $0.33^{\mathrm{a}}$ & $0.35^{\mathrm{ab}}$ & $0.42^{\mathrm{b}}$ & $0.39^{\mathrm{ab}}$ & 0.015 & NS & 0.02 & NS & NS \\
\hline
\end{tabular}


Table 5. continued

\begin{tabular}{|c|c|c|c|c|c|c|c|c|c|}
\hline \multirow{3}{*}{ Fatty acids ${ }^{2}$} & \multirow{2}{*}{\multicolumn{4}{|c|}{ Group }} & \multicolumn{4}{|c|}{ Statistical effect ${ }^{5}$} & \multirow{3}{*}{$\begin{array}{c}\begin{array}{c}\text { Inter- } \\
\text { action }\end{array} \\
\mathrm{OL} \times \mathrm{SE} \\
\mathrm{P} \\
\end{array}$} \\
\hline & & & & & \multicolumn{2}{|c|}{$\mathrm{SE}$} & \multicolumn{2}{|c|}{ LO } & \\
\hline & control & $\mathrm{SE}$ & $\mathrm{LO}$ & $\mathrm{LO}+\mathrm{SE}$ & SEM & $\mathrm{P}$ & SEM & $\mathrm{P}$ & \\
\hline taurine & $0.023^{\mathrm{ab}}$ & $0.026^{\mathrm{a}}$ & $0.031^{\mathrm{b}}$ & $0.025^{\mathrm{a}}$ & 0.001 & NS & 0.001 & NS & NS \\
\hline homo-cysteine & $0.0001^{\mathrm{c}}$ & $0.016^{\mathrm{a}}$ & $0.012^{\mathrm{b}}$ & $0.010^{\mathrm{b}}$ & 0.001 & 0.001 & 0.001 & NS & NS \\
\hline methionine & $0.008^{\mathrm{a}}$ & $0.007^{\mathrm{a}}$ & $0.008^{\mathrm{a}}$ & $0.007^{\mathrm{a}}$ & 0.001 & NS & 0.001 & NS & NS \\
\hline Se-cysteine & $0.0010^{\mathrm{c}}$ & $0.015^{\mathrm{a}}$ & $0.0012^{\mathrm{b}}$ & $0.015^{\mathrm{a}}$ & 0.0002 & 0.001 & 0.002 & 0.001 & 0.01 \\
\hline lysine & $0.031^{\mathrm{a}}$ & $0.030^{\mathrm{a}}$ & $0.042^{\mathrm{b}}$ & $0.037^{\mathrm{b}}$ & 0.002 & NS & 0.003 & NS & NS \\
\hline$\sum \mathrm{S}-\mathrm{AA}^{4}$ & $0.031^{\mathrm{a}}$ & $0.049^{\mathrm{b}}$ & $0.051^{\mathrm{b}}$ & $0.042^{\mathrm{a}}$ & 0.002 & 0.001 & 0.004 & 0.003 & 0.01 \\
\hline$\sum \mathrm{AA}$ & $0.54^{\mathrm{a}}$ & $0.53^{\mathrm{ab}}$ & $0.65^{\mathrm{b}}$ & $0.59^{\mathrm{ab}}$ & 0.026 & NS & 0.034 & NS & NS \\
\hline
\end{tabular}

${ }^{1}$ means in rows with different letters are significantly different at ${ }^{\mathrm{a}, \mathrm{b}} \mathrm{P}<0.05$

${ }^{2}$ abbreviations for fatty acids and other items see Tables 1 and $2 ;{ }^{3}$ the concentration of an assayed compound was below the quantification limit $\left(\mathrm{L}_{\mathrm{Q}}\right) ;{ }^{4}$ the concentration sum of methionine, taurine and homo-cysteine; ${ }^{5} \mathrm{NS}-\mathrm{P}>0.05$

with the control. The perirenal fat tissue and blood plasma CFA was undetectable in these tissues of sheep fed the control diet and the diet enriched in LO. The diet containing LO and SE resulted in a decrease $(\mathrm{P}<0.05)$ in the CFA concentration in the liver and, but not significantly, in M. longissimus dorsi $(\mathrm{P}=0.17)$ compared with the control tissues. There was an LO and SE treatment interaction $(\mathrm{P}<0.01)$ for the CFA concentration in the perirenal fat tissue. The SE supplement decreased $(\mathrm{P}<0.05)$ CFA concentrations in the liver and subcutaneous fat tissue $(\mathrm{P}=0.01)$, whereas they decreased, but not significantly, in M. longissimus dorsi $(\mathrm{P}=0.17)$ and $M$. biceps femoris $(\mathrm{P}=0.41)$.

The influence of dietary LO and SE on the concentrations of monounsaturated fatty acids in tissues of sheep. In the present study, supplemental LO decreased $(\mathrm{P}<0.05)$ the concentration of $c 9 \mathrm{C} 18: 1$ in both muscles, liver and, but not significantly, in the perirenal fat tissue compared with the control. On the other hand, this diet increased $(\mathrm{P}<0.05)$ the $c 9 \mathrm{C} 18: 1$ concentration in the subcutaneous fat tissue and, insignificantly $(\mathrm{P}=0.11)$, in plasma compared with the control. There was an LO and SE treatment interaction for the $c 9 \mathrm{C} 18: 1$ concentration in M. biceps femoris $(\mathrm{P}=0.002)$ and blood plasma $(\mathrm{P}=0.01)$. The diet enriched in $\mathrm{SE}$ decreased the concentration of this acid in M. biceps femoris $(\mathrm{P}<0.05)$, but decreased it, insignificantly however, in the liver and M. longissimus dorsi. On the other hand, this diet resulted in an increase $(\mathrm{P}<0.05)$ in the $c 9 \mathrm{C} 18: 1$ concentration in both fat tissues and blood plasma. Supplemental LO increased the sum of trans octadecenoic acid concentrations $(\Sigma t \mathrm{C} 18: 1)$ in the liver, subcutaneous fat tissue, plasma and, but not significantly, in the perirenal fat tissue compared with the control. The diet enriched in LO resulted in an increase $(\mathrm{P}<0.05)$ in the concentration of MUFA in the fat tissues, plasma, as well as decreased $(\mathrm{P}<0.05)$ the concentration ratio of 
SFA/MUFA in both muscles, the liver, both fat tissues and, but not significantly, in plasma compared with the control. There was an LO and SE treatment interaction for the ratio of SFA/MUFA in the subcutaneous fat tissue $(\mathrm{P}=0.001)$ and plasma $(\mathrm{P}=0.02)$; the diet enriched in $\mathrm{LO}$ and $\mathrm{SE}$ resulted in a decrease of this ratio in all assayed tissues compared with the control group.

The influence of dietary $L O$ and SE on the concentrations of polyunsaturated fatty acids in tissues of sheep. The diets enriched in LO or simultaneously with LO and SE increased $(\mathrm{P}<0.05)$ the concentration of PUFA in the subcutaneous fat tissue, blood plasma and, not significantly, in the perirenal fat $(\mathrm{P}=0.13$ and 0.09 , respectively). Feeding the diet containing LO increased the concentrations $(\mathrm{P}<0.05)$ of $c i s 9$, cis $12 \mathrm{C} 18: 2$ (LA; $c 9, c 12 \mathrm{C} 18: 2)$ and $c 9, c 12, c 15 \mathrm{C} 18: 3$ ( $\alpha$-LNA) in the subcutaneous fat tissue, blood plasma and, not significantly, in both muscles in comparison with the control tissues. On the other hand, the diet enriched in LO, regardless of the presence of SE, decreased $(\mathrm{P}<0.05)$ the concentration of $\alpha$-LNA in the perirenal fat tissue and, not significantly, in the liver. The experimental diets also changed the concentration of $c 6, c 9, c 12 \mathrm{C} 18: 3$ ( $\gamma$-LNA), however, a significant increase $(\mathrm{P}<.05)$ of the $\gamma$-LNA concentration was found in the liver and blood plasma of lambs fed the diet enriched in LO, regardless of the presence of SE. Dietary LO resulted in a decrease $(\mathrm{P}<0.05)$ in the concentration ratio of SFA/PUFA in both muscles and fat tissues, as well as in the liver and blood plasma compared with the control. Similarly, adding SE to the diet enriched in LO decreased the SFA/ PUFA ratio in all assayed tissues compared with the control, however, these changes were in some cases not significant $(\mathrm{P}>0.05)$. The two-factorial interaction between LO and SE was usually significant $(\mathrm{P}<0.05)$ for this ratio of fatty acids in assayed tissues, with the exceptions of the perirenal fat tissue and liver. The diet enriched in SE resulted in an increase $(\mathrm{P}<0.05)$ in the concentration of PUFA in the blood plasma and tended to increase in the perirenal fat tissue $(\mathrm{P}=0.09)$ and subcutaneous fat tissue $(\mathrm{P}=0.08)$ in comparison with the control. This diet decreased the ratio of SFA/PUFA in the liver $(\mathrm{P}<0.05)$, both fat tissues $(\mathrm{P}<0.05)$, M. biceps femoris $(\mathrm{P}<0.05)$, M. longissimus dorsi $(\mathrm{P}=0.07)$, and, but non-significantly, in the blood plasma $(\mathrm{P}=0.21)$.

As can be seen from the data presented in Tables 4 and 5, the diets enriched in $L O$, regardless of the presence of SE, did not significantly change the yield of $\Delta 9$ desaturation in both fat tissues. On the other hand, SE supplementation increased the yield of $\Delta 9$-desaturation in the perirenal fat tissue $(\mathrm{P}<0.05)$ and subcutaneous fat tissue (a tendency: $\mathrm{P}=0.09$ ). As expected, the higher yield of $\Delta 9$-desaturation in both fat tissues resulted in an increase $(\mathrm{P}<0.05)$ in the concentrations of $c 9 \mathrm{C} 18: 1$ and LA in them.

The diet enriched in LO increased $(\mathrm{P}<0.05)$ the concentration sum of all assayed fatty acids $(\Sigma F A)$ in the liver and blood plasma compared with the control. Similarly, 
SE supplementation resulted in a decrease in the $\Sigma F A$ concentration in $M$. biceps femoris $(\mathrm{P}<0.05)$, M. longissimus dorsi $(\mathrm{P}=0.11)$, the liver, both muscles, whereas it increased the concentration of $\Sigma F A$ in blood plasma $(\mathrm{P}<0.05)$.

The influence of dietary $L O$ and $S E$ on the concentrations of amino acids in tissues of sheep. As can be seen from the data summarized in Tables 3, 4 and 5 , the experimental diets generated substantial changes in amino acid (AA) concentrations in both muscles, the liver and blood plasma. AA concentrations in the muscles (Table 3) and liver (Table 4) were analysed in pooled samples prepared by combination of all livers, M. biceps femoris and M. longissimus dorsi from sheep fed the same diet. SE supplementation resulted in an increase in the sum of essential amino acids ( $\Sigma$ E-AA), non-essential amino acids ( $\Sigma$ NE-AA) and sulphur amino acids $(\Sigma \mathrm{S}-\mathrm{AA})$ in both muscles and the liver. On the other hand, this diet showed a negligible influence $(\mathrm{P}>0.05)$ on the concentrations of $\Sigma \mathrm{E}-\mathrm{AA}$, $\Sigma \mathrm{NE}-\mathrm{AA}$ and the sum of all amino acids $(\Sigma \mathrm{AA})$ in blood plasma (Table 5). LO administered to sheep also increased the concentrations of $\Sigma$ E-AA, $\Sigma$ NE-AA and $\Sigma$ S-AA in both muscles (Table 3 ), as well as significantly increasing $\Sigma$ NE-AA, $\Sigma$ SAA and $\Sigma$ AA in the blood plasma, whereas the diet enriched in LO, regardless of the presence of SE, decreased $\Sigma$ E-AA, $\Sigma$ NE-AA, $\Sigma$ S-AA and $\Sigma$ AA in the liver. From the data shown in Tables 3,4 and 5, it results that the concentration of cysteine increased in the muscles of sheep fed the diet enriched in SE and/or LO and decreased in the liver. The concentration of this amino acid was below the quantification limit in blood plasma of sheep fed the control and experimental diets. The diet containing SE increased the concentration of methionine in both muscles, whereas all experimental diets decreased the concentration of this amino acid in the liver. The results presented in Tables 3, 4 and 5 demonstrate that the concentration of lysine in the muscles and liver was slightly increased by SE supplementation, and significantly increased $(\mathrm{P}<0.05)$ in the blood plasma of sheep fed the diets enriched in LO, regardless of the presence of SE.

\section{DISCUSSION}

In the current study and our previous investigations (Czauderna et al., 2004a,b), no macroscopic lesions or toxic symptoms of dietary $5 \% \mathrm{LO}$ and $2 \mathrm{ppm}$ selenium (as selenate) were observed in sheep fed diets enriched in LO and/or SE. Diets containing up to $2 \mathrm{mg}$ selenium per kg would not be toxic for animals, especially for ruminants (Tinggi, 2003; McDowell et al., 2005). Furthermore, in contrast to selenide and selenite, selenate is less reactive and assimilative; consequently, selenate is also less toxic for animals and humans. Therefore, selenium, as selenate, is a significantly better tolerated chemical form of selenium for livestock 
compared with dietary selenite or selenide and seleno-cysteine, in particular. Only chronic dietary inorganic seleno-compounds, especially selenate and selenide, at rates of more than $5 \mathrm{mg}$ Se per $\mathrm{kg}$ can be hepatotoxic and teratogenic in humans and animals (Tapiero et al., 2003; Tinggi, 2003). The $\mathrm{LD}_{50}$ is about $5 \mathrm{mg}$ selenium per kg body weight for animals, thus, this corresponds to about $50 \mathrm{mg} \mathrm{Se} / \mathrm{kg}$.

The present trial demonstrates the ability of supplemented LO to increase BWG of examined sheep as well as the weight of $M$. biceps femoris and M. longissimus dorsi. Moreover, no negative effect on BWG, FCE and weight of the liver and both muscles was found in sheep fed the diet enriched in LO and SE. Values of FCE and BWG summarized in Table 2 document that the diet enriched in LO most efficiently stimulated the efficiency of animal production. Similarly, the diet containing LO and SE effectively increased the body weight of sheep. Thus, our current study is consistent with our previous works (Czauderna et al., 2003b, 2004c; Korniluk et al., 2007) showing that diets enriched in selenium (as organic or inorganic seleno-compound) and a precursor of FA containing conjugated double bonds had a similar effect on FCE and BWG of such monogastric animals as laboratory rats (Czauderna et al., 2003b; Korniluk et al., 2007).

The results show that the experimental diets improved the nutritional quality of M. biceps femoris, M. longissimus dorsi and the liver, as the concentrations of A-SFA, T-SFA in these tissues decreased compared with the controls. A similar effect was found in the perirenal fat tissue when sheep were fed a diet enriched in LO, regardless of the presence of extra selenate. Considering the above results, it can be concluded that these dietary additives possess antiatherogenic properties. Moreover, the diet enriched in LO, regardless of the presence of extra selenate, improved the ratio of SFA/MUFA and SFA/PUFA in both muscles, and in the liver and perirenal fat tissue. As can be seen from the results summarized in Tables 3,4 and 5, improvement in the nutritional value of these tissues correlated with increasing concentrations of MUFA and PUFA in the liver and muscles (Czauderna et al., 2004a,b), both fat tissues, and blood plasma. Selenate supplementation to the LO-enriched diet slightly deteriorated the ratio of SFA/MUFA and SFA/PUFA in both muscles. According to our previous studies (Czauderna et al., 2008), an increase in the ratio of SFA/MUFA and SFA/PUFA in muscles may be related to the higher yield of biohydrogenation in the rumen of sheep fed diets containing selenate. Our recent in vitro studies documented that addition of selenate to ruminal fluids decreased the concentrations of CLA isomers and t11C18:1 in fluids in comparison with the control fluid (Wąsowska et al., 2006). In accordance with our recent studies, a decrease in the concentration of CLA isomers and their metabolites (CFA, i.e.: $c 6, c 9, c 11 C 18: 3, c 6, t 10, c 12 \mathrm{C} 18: 3, c 8, c 11, t 13 \mathrm{C} 20: 3, c 8, t 12, c 14 \mathrm{C} 20: 3$, $c 5, c 8, c 11, t 13 \mathrm{C} 20: 4$ or $c 5, c 8, t 12, c 14 \mathrm{C} 20: 4)$ was found in both muscles and the subcutaneous fat tissue of sheep fed the diet enriched in selenate. Our results 
regarding the abundance of conjugated fatty acids in the liver are also consistent with these findings. Indeed, we found a decrease in the concentration of CLA isomer metabolites in the liver of sheep fed the diet containing selenate. Moreover, the concentration of CLA isomers and their metabolites in this organ of sheep fed the diet enriched in LA and SE was compared with the control group and the group of sheep fed the diet with LO. The increase in CLA isomer concentrations in the liver, $M$. longissimus dorsi, both fat tissues, and blood plasma led us to suggest that the yield of the biohydrogenation of formed CLA isomers in the rumen was reduced by LO supplemented to the diet compared with the control sheep. Addition of Se to the diet enriched in LO elevated the yield of biohydrogenation of CLA isomers in the rumen compared with sheep fed the diet containing LO.

As can be seen from data summarized in Tables 3, 4 and 5 and our recent studies, LO supplementation resulted in a decrease in $\Delta 9$-desaturation in both muscles, the liver, blood plasma and subcutaneous fat tissue (Czauderna et al., 2004a,b). Considering the above, it can be concluded that unsaturated fatty acids contained in dietary LO lowered the yield of desaturation of FA in tissues of sheep compared with the control animals. SE added to the diet with LO slightly elevated the yield of $\Delta 9$-desaturation (a positive interaction) in the tissues of sheep compared with sheep fed the diet enriched in LO. In accordance with these results, we found that selenate supplementation increased the yield of $\Delta 9$-desaturation in all assayed tissues (Czauderna et al., 2004a,b) compared with sheep fed the diet containing only LO.

The results from this study indicate that the $\Sigma$ S-AA concentration in $M$. biceps femoris, M. longissimus dorsi and blood plasma increased with LO and/ or SE supplementation. The finding that these diets fed to sheep increased the concentrations of $\Sigma \mathrm{E}-\mathrm{AA}$, methionine and taurine in both muscles (Table 3) is valuable for nutritionists in the context of research on improving the nutritional quality of meat of ruminants for human health.

\section{CONCLUSIONS}

Feeds enriched in linseed oil, regardless of the presence of selenate, can be permanently used to increase the concentration of fatty acids containing conjugated double bonds (e.g., CLA isomers) in tissues of ruminants without adversely influencing performance. The experimental diets enriched in LA with or without selenate increased the nutritional value of $M$. biceps femoris and $M$. longissimus dorsi as the ratio of SFA/MUFA and SFA/PUFA decreased, as well as increased the concentration of essential amino acids (i.e.: histidine, valine, leucine, iso-leucine, lysine, methionine, threonine and phenylalanine). Moreover, 
the diet containing selenate, regardless of the presence of LO, resulted in a $\sim 2$-fold increase in the Se concentration in M. biceps femoris, $M$. longissimus dorsi, blood plasma and $\sim 10$-fold in the liver of sheep compared with the control animals. Therefore, the results of our short-term study constitute important information for nutritionists carrying out further investigations to improve the nutritional quality of feed for ruminants and humans. On the other hand, further research is necessary to determine if dietary selenate and other vegetable oils induce changes in profiles of fatty acids and amino acids in ruminant meat that are more beneficial to human health.

\section{REFERENCES}

Crespo A.M., Reis M.A., Lanca M.J., 1995. Effect of selenium supplementation on poly-unsaturated fatty acids in rats. Biol. Tr. Elem. Res. 47, 335-341

Czauderna M., Kowalczyk J., 2002. HPLC separation of some unsaturated and saturated fatty acids. Chem. Anal. (Warsaw) 47, 867-882

Czauderna M., Kowalczyk J., Niedźwiedzka K.M., Wallace R.J., 2008. Influence of selenite, selenate and $\alpha$-linolenic acid on the fatty acid profile in in vitro incubated ovine ruminal fluid. Proc. Soc. Nutr. Physiol. 17, 69

Czauderna M., Kowalczyk J., Niedźwiedzka K.M., Wąsowska I., 2002a. Determination of free- and protein primary amino acids in biological materials by high-performance liquid chromatography and photodiode array detection. J. Anim. Feed Sci. 11, 143-167

Czauderna M., Kowalczyk J., Niedźwiedzka K.M., Wąsowska I., Pająk J.J., 2004b. The effect of selenium and linseed oil on growth of sheep and content of selected fatty acids in M. longissimus dorsi. J. Anim. Feed Sci. 13, Suppl. 1, 303-306

Czauderna M., Kowalczyk J., Niedźwiedzka K.M., Wąsowska I., Pająk J.J., Bulska E., Ruszczyńska A., 2004a. The effect of linseed oil and selenium on the content of fatty acids and some elements in the liver and selected tissues of sheep. J. Anim. Feed Sci. 13, Suppl. 2, 103-106

Czauderna M., Kowalczyk J., Niedźwiedzka K.M., Wąsowska I., Pastuszewska B., 2004c. Conjugated linoleic acid (CLA) content and fatty acids composition of muscle in rats fed isomers of CLA and selenium. J. Anim. Feed Sci. 13, 183-196

Czauderna M., Kowalczyk J., Wąsowska I., Niedźwiedzka K.M., 2002b. A highly efficient method for derivatization of fatty acids for high performance liquid chromatography. J. Anim. Feed Sci. $11,517-526$

Czauderna M., Kowalczyk J., Wąsowska I., Niedźwiedzka K.M., 2003a. Determination of conjugated linoleic acid isomers by liquid chromatography and photodiode array detection. J. Anim. Feed Sci. 12, 269-382

Czauderna M., Kowalczyk J., Wąsowska I., Niedźwiedzka K.M., Pastuszewska B., 2003b. The effects of selenium and conjugated linoleic acid (CLA) isomers on fatty acid composition, CLA isomer content in tissues, and growth of rats. J. Anim. Feed Sci. 12, 865-881

Flachowsky G., Erdmann K., Hüther L., Jahreis G., Möckel P., Lebzien P., 2006. Influence of roughage/concentrate ratio and linseed oil on the concentration of trans-fatty acids and conjugated linoleic acid in duodenal chyme and milk fat of late lacting cows. Arch. Anim. Nutr. $60,501-511$ 
Korniluk K., Czauderna M., Kowalczyk J., 2007. The influence of dietary conjugated linoleic acid isomers and high-selenized yeast on the fatty acid profile of spleen, pancreas and kidneys of rats. J. Anim. Feed Sci. 16, 121-139

McDowell L.R., Davis P.A., Cristaldi L.A., Wilkinson N.S., Buergelt C.D., Van Alstyne R., 2005. Toxicity of selenium: fear or precaution? Feedstuffs 77 (22), 12-13

Murphy K.J., Meyer B., Mori T.A., Burke V., Mansour J., Patch C.S., Tapsell L.C., Noakes M., Clifton P.A., Barden A., Puddey I.B., Beilin L.J., Howe P.R.C., 2007. Impact of foods enriched with n-3 long-chain polyunsaturated fatty acids on erythrocyte n-3 levels and cardiovascular risk factors. Brit. J. Nutr. 97, 749-757

Niedźwiedzka K.M., Wąsowska I., Czauderna M., Kowalczyk J., Pastuszewska B., 2006. Influence of dietary conjugated linoleic acid isomers and Se on fatty acids profile in blood plasma and some tissues of rats. J. Anim. Feed Sci. 15, 471-489

Raes K., De Smet S., Demeyer D., 2004. Effect of dietary fatty acids on incorporation of long chain polyunsaturated fatty acids and conjugated linoleic acid in lamb, beef and pork meat: a review. Anim. Feed Sci. Tech. 113, 199-221

Schweizer U., Streckfub F., Pelt P., Carlson B.A., Hatfield D.L., Köhrle J., Schomburg L., 2005. Hepatically derived selenoprotein $\mathrm{P}$ is a key factor to kidney but not for brain selenium supply. Biochem. J. 386, 221-226

Statistica by StatSoft, 2002. Web: www.statsoft.pl

Tanguy S., Besse S., Ducros V., Leiris J., Boucher F., 2003. Effect of increased dietary selenium intake on blood and cardiac selenium status during aging in rats. Nutr. Res. 23, 239-243

Tapiero H., Townsend D.M., Tew K.D., 2003. The antioxidant role of selenium and selenocompounds. Biomed. Pharmacotherapy 57, 134-144

Tinggi U., 2003. Essentiality and toxicity of selenium and its status in Australia: A review. Toxicol. Lett. 137, 103-110

Wąsowska I., Czauderna M., Kowalczyk J., Wallace R.J., 2006. Impact of selenite and selenate on conjugated linoleic acid isomers in incubated ruminal fluid. J. Anim. Feed Sci. 15, Suppl. 1, 97-100

Yu L.L., Wang R.L., Zhang Y.Z., Kleemann D.O., Zhu X.P., Jia Z.H., 2008. Effects of selenium supplementation on polyunsaturated fatty acid concentrations and antioxidant status in plasma and liver of lambs fed linseed oil or sunflower oil diets. Anim. Feed Sci. Tech. 140, 39-51 